\title{
Diagnostic performance of rapid diagnostic test, light microscopy and polymerase chain reaction during mass survey conducted in low and high malaria-endemic areas from two North-Eastern states of India
}

\author{
Hari Shankar ${ }^{1} \cdot$ Mrigendra Pal Singh $^{1} \cdot$ Sobhan Phookan ${ }^{2} \cdot$ Kuldeep Singh $^{2} \cdot$ Neelima Mishra $^{1}$ (D) \\ Received: 17 December 2020 / Accepted: 15 March 2021 / Published online: 27 March 2021 \\ (C) The Author(s), under exclusive licence to Springer-Verlag GmbH Germany, part of Springer Nature 2021
}

\begin{abstract}
An early and accurate diagnosis followed by prompt treatment is pre-requisite for the management of any disease. Malaria diagnosis is routinely performed by microscopy and rapid diagnostic tests (RDTs) in the field settings; however, their performance may vary across regions, age and asymptomatic status. Owing to this, we assessed the diagnostic performance of conventional and advanced molecular tools for malaria detection in low and high malaria-endemic settings. We performed mass blood surveys in low and high endemic regions of two North-Eastern districts from the states of Assam and Meghalaya. A total of 3322 individuals were screened for malaria using RDT, microscopy and PCR and measures of diagnostic accuracy were estimated. Out of 3322 individuals, 649 (19.5\%) were detected with malaria parasite. Asymptomatic were $86.4 \%$ (2872/3322), of which 19.4\% (557/2872) had Plasmodium infection. The sensitivity and specificity of microscopy were $42.7 \%$ and $99.3 \%$, and RDT showed $49.9 \%$ and $90.4 \%$, respectively, considering PCR as standard. RDT (AUC: 0.65 vs 0.74 ; $p=0.001$ ) and microscopy (AUC: 0.64 vs $0.76 ; p<0.0001$ ) performances were significantly lower in low compared to high endemic areas. True positive rate was lower in asymptomatics but true negative rate was found similar to symptomatic individuals. The conventional diagnostic tools (RDT and microscopy) had detected malaria in children with nearly twofold greater sensitivity than in the adults $(p<0.05)$. To conclude, asymptomatics, adults and low malaria-endemic regions require major attention due to mediocre performance of conventional diagnostic tools in malaria detection.
\end{abstract}

Keywords Asymptomatic $\cdot$ Diagnosis $\cdot$ Endemicity $\cdot$ Malaria $\cdot$ Molecular method

\section{Introduction}

According to World Malaria Report 2019, WHO estimated 228 million malaria cases in 2018 and 231 million cases in 2017 worldwide and reported that nineteen countries in subSaharan Africa and India accounted for nearly $85 \%$ of the global malaria cases in 2018 (WHO World Malaria Report

Section Editor: Nawal Hijjawi

Neelima Mishra

neelima1.nimr@gmail.com

1 Parasite-Host Biology Group, ICMR-National Institute of Malaria Research, Sector-8, Dwarka, New Delhi 110077, India

2 ICMR-National Institute of Malaria Research Field Unit, Guwahati, Assam 781022, India
2019). Despite the achievements in reducing the malaria burden, there are still substantial numbers of malaria cases across the globe causing morbidity and mortality. In recent years, due to the pace of malaria elimination programme in India, a downfall trend in number of malaria cases and deaths has been observed (National Vector Borne Disease Control Programme 2019). To maintain this pace, accurate diagnosis and prompt treatment of malaria cases is of utmost priority so that even the last parasite circulating in the community can be detected and eliminated.

In the current scenario, microscopy and rapid diagnostic tests (RDTs) are being used for malaria diagnosis (WHO World Malaria Report 2019), where microscopy is considered gold standard. Indeed, remote tribal areas and low resource settings are more inclined towards using RDTs in comparison to microscopy, due to its user-friendly operations, quick results and no requirements of any equipment, electricity or 
technical speciality whatsoever (McMorrow et al. 2011). Recent evidence suggests the presence of sub-microscopic infections (van Eijk et al. 2019), low-density Plasmodium infection and asymptomatic parasitaemia in Indian community (van Eijk et al. 2020; Shankar et al. 2021), even in low transmission settings (Kaura et al. 2019). The presence of such hidden reservoir of infectiousness may pose a challenge to the malaria elimination programme and therefore must be curtailed to achieve the elimination goal. Nevertheless, India has shown its strong commitment by developing the National Framework for Malaria Elimination, in which the states as well as districts are categorised based on annual parasite incidence and district wise malaria confinement and elimination strategies have been devised (Ministry of Health, and Family Welfare, Government of India 2016). These strategies however would be more effective if should be applied in conjunction with more sensitive and accurate diagnostic methods especially in area-specific manner, i.e. using advanced molecular tools in areas reported to have low-density Plasmodium infections.

Malaria diagnosis always remains a grey area of research despite knowing its importance in disease management. Thus in addition to antimalarial drug and vaccine development research, there is a need to invest and focus on improving the utility of existing diagnostics and developing new methods for accurate detection of parasites (Wongsrichanalai et al. 2007). This would not only prevent misdiagnosis leading to the widespread overtreatment with antimalarials, which is one of the factors responsible for the development of drug resistance in Plasmodium, but will also reduce the cost incurred on antimalarial drug treatment. Moreover, related deaths can be averted if the patients receive accurate malaria diagnosis and correct treatment (Chanda et al. 2009; Uzochukwu et al. 2009). It is obvious that a reliable diagnostic test with high sensitivity and specificity can avert malaria-related deaths, increasing drug resistance, unnecessary treatments and rising drug costs (Rafael et al. 2006). Therefore, field evaluation of diagnostic accuracy of currently used malaria detection methods (microscopy and RDT) should be performed. In this study, we performed mass surveys in high and low malaria-endemic areas to evaluate the diagnostic performance of microscopy, RDT and PCR among different age groups and asymptomatic individuals from two North-Eastern districts of India.

\section{Materials and methods}

The present study is derived from a subset of data obtained by conducting mass blood surveys during dry season, i.e. between February to April 2017, in two North-Eastern districts from the states of Assam and Meghalaya of India. During the surveys, a total of 9118 individuals were screened for malaria by on-spot diagnosis using SD Bioline malaria Ag pf/pv RDT which detects histidine-rich protein 2 (HRP2) antigen specific to Plasmodium falciparum and Plasmodium lactate dehydrogenase (pLDH) specific to Plasmodium vivax and light microscopy in the field laboratory. Based on the RDT results, the individuals were given antimalarial treatment as per the National guidelines for the treatment of malaria in India. Dried blood spot (DBS) on Whatman filter paper, grade 3 CHR (Merck, Germany, UK) was prepared for performing parasite genotyping using polymerase chain reaction (PCR) in the test laboratory. The individuals of all age groups and gender irrespective of any clinical sign and symptoms of malaria were included in the study. Out of total dataset of 9118 samples, 3500 were drawn randomly for diagnosis using PCR, of which 43 samples could not be amplified for PCR analysis. By doing this random selection, each sample had equal chances of being selected for PCR diagnosis. Furthermore, we performed power analysis by considering $90 \%$ power and 5\% alpha and found that the sample size of subset was large enough to generalise the findings. A subset of samples ( $n$ $=3322$ ) diagnosed with all three methods, i.e. RDT, microscopy and PCR, were included in this study to estimate their diagnostic accuracy stratified by endemicity, symptoms and age of the participants. The flow diagram showing population screening and diagnosis performed is shown in Fig. 1.

Study districts were categorised in low and high malariaendemic regions based on the reported malaria incidence rate during the last 5 years preceding the survey. Asymptomatic cases were defined as participants who have neither reported history of a fever during 2 weeks preceding the survey nor having body temperature $\geq 37.5^{\circ} \mathrm{C}$ at the time of examination. Axillary body temperature was recorded using a digital thermometer at the time of sample collection. The participants were categorised as child who were 14 or less years of age.

\section{Microscopic examination}

Thick and thin blood smears were prepared at the study site and transported daily to the local laboratory, where fixation of thin blood smears was performed with methanol followed by staining of both thick and thin smears with 3\% Giemsa stain solution for $30 \mathrm{~min}$. All the slides were examined under oil emersion $(10 \times 100$ magnification) by experienced microscopists. Thick films were used to identify the presence of parasites, whereas thin films were used to identify species of malaria parasites. A blood slide was considered negative if no parasites were found after examining 100 microscopic fields. For all positive slides, malaria species identification was done. All the microscopists were blinded for RDT results, and later the discordant results (RDT ${ }^{+v e}$ Microscopy ${ }^{-v e}$ and RDT $^{-v e}$ Microscopy ${ }^{+\mathrm{ve}}$ ) were re-examined by another experienced microscopist and/or a WHO certified microscopist who was not aware of previous microscopy/RDT results. The final microscopy results were considered positive or negative depending upon the quality check results of the microscopy. 
Fig. 1 Flow diagram showing population screening and diagnosis performed

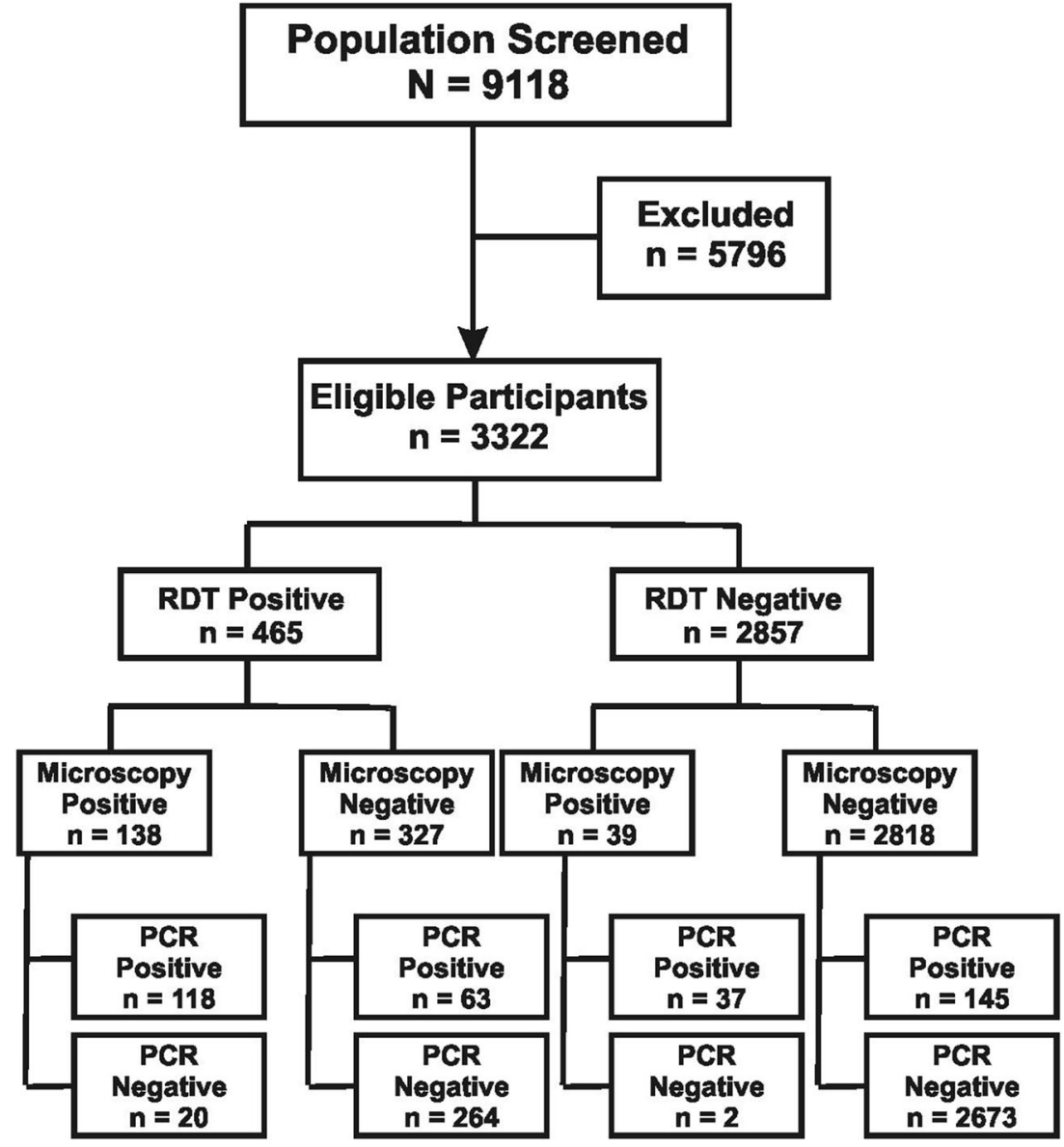

\section{DNA extraction from DBS}

Genomic DNA was isolated from DBS using the QIAamp Blood Mini Kit (QIAGEN, USA) according to the manufacturer's protocol. Three punched-out circles ( $3 \mathrm{~mm}$ each) from DBS were placed in a $1.5-\mathrm{ml}$ microcentrifuge tube and incubated overnight at room temperature with $180 \mu$ buffer. The subsequent genomic DNA extraction steps were followed as mentioned in the instruction leaflet (Qiagen 2020).

\section{Detection of Plasmodium species using PCR}

The PCR protocol followed in this study to identify Plasmodium species using PCR is described elsewhere (Snounou et al. 1993), which was based on targeting coding sequences of the small subunit of ribosomal RNA specific to the parasite species with the detection limit ranged between 1 and 10 parasite per microliter of blood. The primers used in this study were detailed below:

$\left\{\begin{array}{c}\text { rPF1 5'-AGAAATAGAGTAAAAAACAATTTA-3' } \\ \text { rPF2 5'-GTAACTATTCTAGGGGAACTA-3' }\end{array}\right.$ $\left\{\right.$ rPV1 5'-CCGAATTCAGTCCCACGT- $3^{\prime}$

$\{$ rPV2 5'-GCTTCGGC'I-FGGAAGTCC-3'

$\left\{\begin{array}{c}\text { rPM1 5'-CGTACAACTATGTTATAAATATACAA-3' } \\ \text { rPM2 5'-CTCTTAATTCTTATGTTTTT-3 }{ }^{\prime}\end{array}\right.$

$\{$ rPO1 5'-CACATTAATTGTATCCTAGTG-3'

\{rPLU6 5'-TTAAAATTGTTGCAGTTAAAACG-3'

Briefly, $25 \mu \mathrm{l}$ reaction mixtures consisted of $12.5 \mu \mathrm{l}$ DreamTaq Green PCR mix, $0 \cdot 4 \mu \mathrm{M}$ of each primer (10 uM stock), $2 \mu$ l of DNA and added sterile water up to $25 \mu \mathrm{l}$. All PCR amplifications were carried-out in Applied Biosystem thermocycler as follows: $5 \mathrm{~min}$ at $95{ }^{\circ} \mathrm{C}$, followed by 35 cycles of $30 \mathrm{~s}$ at $95{ }^{\circ} \mathrm{C}, 30 \mathrm{~s}$ at $56^{\circ} \mathrm{C}$ and $1 \mathrm{~min}$ at $72{ }^{\circ} \mathrm{C}$, with a final extension of $7 \mathrm{~min}$ at $72^{\circ} \mathrm{C}$. PCR amplifications were analysed on $2 \%$ agarose gels prepared with $0.5 \mathrm{X}$ Tris-Borateethylenediaminetetraacetic acid buffer in the presence of ethidium bromide. After obtaining the PCR results, the discordant samples $\left(\mathrm{RDT}^{+\mathrm{ve}} \mathrm{Microscopic}^{+\mathrm{ve}} \mathrm{PCR}^{-\mathrm{ve}}\right.$ and $\mathrm{RDT}^{-\mathrm{ve}}$ Microscopic $^{-\mathrm{ve}} \mathrm{PCR}^{+\mathrm{ve}}$ ) were re-confirmed using light microscopy and PCR. 
Table 1 Diagnostic performance of microscopy, RDT and PCR in mass blood surveys $(n=3322)$

\begin{tabular}{|c|c|c|c|c|c|c|}
\hline \multirow[t]{2}{*}{ Diagnostics used } & \multicolumn{2}{|c|}{ PCR as standard } & \multicolumn{2}{|c|}{$\begin{array}{l}\text { Microscopy as } \\
\text { standard }\end{array}$} & \multicolumn{2}{|c|}{ RDT as standard } \\
\hline & Positive & Negative & Positive & Negative & Positive & Negative \\
\hline \multicolumn{7}{|l|}{ A. Microscopy } \\
\hline Positive & 155 & 22 & & & 138 & 39 \\
\hline Negative & 208 & 2937 & & & 327 & 2818 \\
\hline Sensitivity $(95 \% \mathrm{CI})$ & \multicolumn{2}{|c|}{$42.7(37.6-48.0)$} & & & \multicolumn{2}{|c|}{$29.7(25.6-34.1)$} \\
\hline Specificity $(95 \% \mathrm{CI})$ & \multicolumn{2}{|c|}{$99.3(98.9-99.5)$} & & & \multicolumn{2}{|c|}{$98.6(98.1-99.0)$} \\
\hline Positive predictive value $(95 \% \mathrm{CI})$ & \multicolumn{2}{|c|}{$87.6(81.8-92.0)$} & & & \multicolumn{2}{|c|}{$78.0(71.1-83.8)$} \\
\hline Negative predictive value $(95 \% \mathrm{CI})$ & \multicolumn{2}{|c|}{$93.4(92.5-94.2)$} & & & \multicolumn{2}{|c|}{$89.6(88.5-90.6)$} \\
\hline Accuracy $(95 \% \mathrm{CI})$ & \multicolumn{2}{|c|}{$93.1(92.2-93.9)$} & & & \multicolumn{2}{|c|}{$89.0(87.9-90.0)$} \\
\hline Youden's $J$ index $(95 \%$ CI) & \multicolumn{2}{|c|}{$0.42(0.36-0.47)$} & & & \multicolumn{2}{|c|}{$0.28(0.24-0.33)$} \\
\hline \multicolumn{7}{|l|}{ B. RDT } \\
\hline Positive & 181 & 284 & 138 & 327 & & \\
\hline Negative & 182 & 2675 & 39 & 2818 & & \\
\hline $\begin{array}{l}\text { Sensitivity }(95 \% \text { CI) } \\
\text { Specificity }(95 \% \text { CI) }\end{array}$ & \multicolumn{2}{|c|}{$\begin{array}{l}49.9(44.6-55.1) \\
90.4(89.3-91.4)\end{array}$} & $\begin{array}{l}78.0(71 . \\
89.6(88 .\end{array}$ & $\begin{array}{l}83.8) \\
-90.6)\end{array}$ & & \\
\hline Positive predictive value $(95 \% \mathrm{CI})$ & \multicolumn{2}{|c|}{$38.9(34.5-43.5)$} & \multicolumn{2}{|c|}{$29.7(25.6-34.1)$} & & \\
\hline Negative predictive value $(95 \% \mathrm{CI})$ & \multicolumn{2}{|c|}{$93.6(92.7-94.5)$} & \multicolumn{2}{|c|}{$98.6(98.1-99.0)$} & & \\
\hline Accuracy $(95 \% \mathrm{CI})$ & \multicolumn{2}{|c|}{$86.0(84.8-87.1)$} & \multicolumn{2}{|c|}{$89.0(87.9-90.0)$} & & \\
\hline Youden's $J$ index $(95 \% \mathrm{CI})$ & \multicolumn{2}{|c|}{$0.40(0.34-0.47)$} & \multicolumn{2}{|c|}{$0.68(0.60-0.74)$} & & \\
\hline \multicolumn{7}{|l|}{ C. PCR } \\
\hline Positive & & & 155 & 208 & 181 & 182 \\
\hline Negative & & & 22 & 2937 & 284 & 2675 \\
\hline Sensitivity $(95 \% \mathrm{CI})$ & & & \multicolumn{2}{|c|}{$87.6(81.8-92.0)$} & \multicolumn{2}{|c|}{$38.9(34.5-43.5)$} \\
\hline Specificity $(95 \%$ CI) & & & \multicolumn{2}{|c|}{$93.4(92.5-94.2)$} & $93.6(92$ & $-94.5)$ \\
\hline Positive predictive value $(95 \% \mathrm{CI})$ & & & $42.7(37$ & $48.0)$ & $49.9(44$ & $-55.1)$ \\
\hline Negative predictive value $(95 \% \mathrm{CI})$ & & & $99.3(98$. & $-99.5)$ & $90.4(89$ & $-91.4)$ \\
\hline Accuracy $(95 \% \mathrm{CI})$ & & & $93.1(92$. & -93.9) & $86.0(84$ & $-87.1)$ \\
\hline Youden's $J$ index $(95 \% \mathrm{CI})$ & & & $0.81(0.7$ & $-0.86)$ & $0.33(0.2$ & $-0.38)$ \\
\hline
\end{tabular}

\section{Data analysis}

The data were entered into Microsoft Excel 2007 worksheet and cross-checked for typographical errors. Furthermore, lists of quality checks have been applied in order to ensure quality of data. Data was analysed using R 3.4.3 for Windows (R Project for Statistical Computing). Diagnostic performance of RDT, microscopy and PCR was expressed in terms of sensitivity, specificity, positive and negative predictive values (PPV and NPV, respectively). The epi.tests module of epiR package (Package epiR version 1.0-14, available at: https:// cran.r-project.org/web/packages/epiR/epiR.pdf) was used to calculate diagnostic performance. Receiver operating characteristic (ROC) curve tool was used for diagnostic test evaluation. A $p$-value $<0 \cdot 05$ was considered statistically significant.

\section{Results}

A total of 3322 individuals were screened for malaria using RDT, microscopy and PCR, of which 1889 (56.9\%) were from high and $1433(43.1 \%)$ from low endemic regions. Mean age of participants was $17.2 \pm 15.7$ years; $63.4 \%$ were children (14 years or below age group) and $86.4 \%$ (2872/3322) were asymptomatic of which $19.4 \%$ (557/2872) had Plasmodium infection irrespective of the diagnostic method used in this study.

The estimates of sensitivity, specificity, PPV, NPV, accuracy, Youden's $J$ index of microscopy and RDT were 42.7, 99.3, 87.6, 93.4, 93.1, 0.42 and 49.9, 90.4, 38.9, 93.6, 86.0, 0.40 , respectively, when PCR was considered a reference standard (Table 1). When adopting microscopy as a reference, such measures of RDT and PCR were 78.0, 89.6, 29.7, 98.6, 89.0, 0.68 and 87.6, 93.4, 42.7, 99.3, 93.1, 0.81, respectively. 
Similarly, measures of sensitivity, specificity, PPV, NPV, accuracy, Youden's $J$ index of microscopy and PCR were 29.7, 98.6, 78.0, 89.6, 89.0, 0.28 and 38.9, 93.6, 49.9, 90.4, 86.0, 0.33 respectively considering RDT as a reference standard (Table 1). Measures used to check the performance of malaria diagnostic tools in low and high endemic regions revealed drastically lower performance of microscopy in low endemic regions (Youden's $J$ index: 0.28 vs 0.52 ) but PCR performance differs slightly between low and high endemic regions (Youden's $J$ index: 0.74 vs 0.85 ). Though, RDT showed lower sensitivity ( $37.8 \%$ vs $58.9 \%)$, Youden's $J$ index ( 0.31 vs 0.47 ) and higher specificity ( $92.8 \%$ vs $88.6 \%$ ) in low compared to high endemic regions while using PCR as a reference, but a reverse scenario was observed when microscopy was adopted as a reference standard (Table 2). The results suggest improved malaria detection ability of diagnostic tools in symptomatic patients compared to asymptomatic individuals, and PCR was highly efficient in malaria detection followed by RDT, irrespective of symptomatic status (Table 3). Measures of sensitivity, specificity, PPV, NPV, accuracy and Youden's $J$ index demonstrated better case detection ability of all three malaria diagnostic tools, viz. RDT, microscopy and PCR in children compared to adults (Table 4).

ROC curve analysis revealed that area under curve (AUC) for RDT and microscopy did not differ significantly against PCR as a reference standard. Furthermore, plotting of AUC for RDT and microscopy by endemicity, symptoms and age group showed improved performance of RDT (AUC: 0.74 vs $0.65 ; p=0.001$ ) and microscopy (AUC: 0.76 vs $0.64 ; p<0.0001$ ) in high compared to

Table 2 Comparison of diagnostic accuracy of microscopy, RDT and PCR in low and high endemic regions

\begin{tabular}{|c|c|c|c|c|c|c|c|c|c|c|c|c|}
\hline \multirow{3}{*}{ Diagnostics used } & \multicolumn{6}{|c|}{ Low endemic regions $(n=1433)$} & \multicolumn{6}{|c|}{ High endemic regions $(n=1889)$} \\
\hline & \multicolumn{2}{|c|}{ PCR (standard) } & \multicolumn{2}{|c|}{$\begin{array}{l}\text { Microscopy } \\
\text { (standard) }\end{array}$} & \multicolumn{2}{|c|}{ RDT (standard) } & \multicolumn{2}{|c|}{ PCR (standard) } & \multicolumn{2}{|c|}{ Microscopy (standard) } & \multicolumn{2}{|c|}{ RDT (standard) } \\
\hline & $+\mathrm{ve}$ & $-\mathrm{ve}$ & +ve & $-\mathrm{ve}$ & $+\mathrm{ve}$ & $-\mathrm{ve}$ & $+\mathrm{ve}$ & $-\mathrm{ve}$ & $+\mathrm{ve}$ & $-\mathrm{ve}$ & $+\mathrm{ve}$ & $-\mathrm{ve}$ \\
\hline \multicolumn{13}{|l|}{ A. Microscopy } \\
\hline Positive & 45 & 10 & & & 46 & 9 & 110 & 12 & & & 92 & 30 \\
\hline Negative & 111 & 1267 & & & 105 & 1273 & 97 & 1670 & & & 222 & 1545 \\
\hline Sensitivity $(95 \% \mathrm{CI})$ & \multicolumn{4}{|c|}{$28.8(21.9-36.6)$} & \multicolumn{2}{|c|}{$30.5(23.2-38.5)$} & \multicolumn{4}{|c|}{$53.1(46.1-60.1)$} & \multicolumn{2}{|c|}{$29.3(24.3-34.7)$} \\
\hline Specificity $(95 \% \mathrm{CI})$ & \multicolumn{4}{|c|}{$99.2(98.6-99.6)$} & \multicolumn{2}{|c|}{$99.3(98.7-99.7)$} & \multicolumn{4}{|c|}{$99.3(98.8-99.6)$} & \multicolumn{2}{|c|}{$98.1(97.3-98.7)$} \\
\hline PPV $(95 \% \mathrm{CI})$ & \multicolumn{4}{|c|}{$81.8(69.1-90.9)$} & \multicolumn{2}{|c|}{$83.6(71.2-92.2)$} & \multicolumn{4}{|c|}{$90.2(83.4-94.8)$} & \multicolumn{2}{|c|}{$75.4(66.8-82.8)$} \\
\hline NPV $(95 \%$ CI) & \multicolumn{4}{|c|}{$91.9(90.4-93.3)$} & \multicolumn{2}{|c|}{$92.4(90.9-93.7)$} & \multicolumn{4}{|c|}{$94.5(93.3-95.5)$} & \multicolumn{2}{|c|}{$87.4(85.8-88.9)$} \\
\hline Accuracy $(95 \% \mathrm{CI})$ & \multicolumn{4}{|c|}{$91.6(90.1-93.0)$} & \multicolumn{2}{|c|}{$92.0(90.6-93.4)$} & 94.2 & $3.2-95.3)$ & & & 86.7 & $5.1-88.2)$ \\
\hline Youden's $J$ index $(95 \% \mathrm{CI})$ & 0.28 & $.20-0.36)$ & & & 0.30 & $.22-0.38)$ & 0.52 & $.45-0.60)$ & & & 0.27 & $.22-0.33)$ \\
\hline B. RDT & & & & & & & & & & & & \\
\hline Positive & 59 & 92 & 46 & 105 & & & 122 & 192 & 92 & 222 & & \\
\hline Negative & 97 & 1185 & 9 & 1273 & & & 85 & 1490 & 30 & 1545 & & \\
\hline Sensitivity (95\% CI) & 37.8 & $0.2-45.9)$ & 83.6 & $1.2-92.2)$ & & & 58.9 & $1.9-65.7)$ & 75.4 & $-82.8)$ & & \\
\hline Specificity $(95 \%$ CI) & 92.8 & $1.2-94.2)$ & 92.4 & $0.9-93.7)$ & & & 88.6 & $7.0-90.1)$ & 87.4 & $-88.9)$ & & \\
\hline PPV (95\% CI) & 39.1 & $1.2-47.3)$ & 30.5 & $3.2-38.5)$ & & & 38.9 & $3.4-44.5)$ & 29.3 & $-34.7)$ & & \\
\hline NPV (95\% CI) & 92.4 & $0.8-93.8)$ & 99.3 & 8.7-99.7) & & & 94.6 & 3.4-95.7) & 98.1 & $-98.7)$ & & \\
\hline Accuracy $(95 \% \mathrm{CI})$ & 86.8 & $5.1-88.6)$ & 92.0 & $0.6-93.4)$ & & & 85.3 & $3.7-86.9)$ & 86.7 & $-88.2)$ & & \\
\hline Youden's $J$ index $(95 \% \mathrm{CI})$ & 0.31 & $.21-0.40)$ & 0.76 & $.62-0.86)$ & & & 0.47 & $.39-0.56)$ & 0.63 & $-0.72)$ & & \\
\hline C. PCR & & & & & & & & & & & & \\
\hline Positive & & & 45 & 111 & 59 & 97 & & & 110 & 97 & 122 & 85 \\
\hline Negative & & & 10 & 1267 & 92 & 1185 & & & 12 & 1670 & 192 & 1490 \\
\hline Sensitivity $(95 \% \mathrm{CI})$ & & & 81.8 & $9.1-90.9)$ & 39.1 & $1.2-47.3)$ & & & 90.2 & $-94.8)$ & 38.9 & $3.4-44.5)$ \\
\hline Specificity $(95 \% \mathrm{CI})$ & & & 91.9 & $0.4-93.3)$ & 92.4 & $0.8-93.8)$ & & & 94.5 & $-95.5)$ & 94.6 & $3.4-95.7)$ \\
\hline PPV $(95 \% \mathrm{CI})$ & & & 28.8 & $1.9-36.6)$ & 37.8 & $0.2-45.9)$ & & & 53.1 & $-60.1)$ & 58.9 & $1.9-65.7)$ \\
\hline NPV (95\% CI) & & & 99.2 & $8.6-99.6)$ & 92.8 & $1.2-94.2)$ & & & 99.3 & $-99.6)$ & 88.6 & $7.0-90.1)$ \\
\hline Accuracy $(95 \% \mathrm{CI})$ & & & 91.6 & $0.1-93.0)$ & 86.8 & $5.1-88.6)$ & & & 94.2 & $-95.3)$ & 85.3 & $3.7-86.9)$ \\
\hline Youden's $J$ index $(95 \% \mathrm{CI})$ & & & 0.74 & $.59-0.84)$ & 0.31 & $.22-0.41)$ & & & 0.85 & $-0.90)$ & 0.33 & $.27-0.40)$ \\
\hline
\end{tabular}

$P P V$, positive predictive value; $N P V$, negative predictive value; $+v e$, positive; $-v e$, negative 
Table 3 Diagnostic performance of microscopy, RDT and PCR in asymptomatic and symptomatic individuals

\begin{tabular}{|c|c|c|c|c|c|c|c|c|c|c|c|c|}
\hline \multirow{3}{*}{ Diagnostics used } & \multicolumn{6}{|c|}{ Asymptomatic $(n=2872)$} & \multicolumn{6}{|c|}{ Symptomatic $(n=450)$} \\
\hline & \multicolumn{2}{|c|}{ PCR (standard) } & \multicolumn{2}{|c|}{$\begin{array}{l}\text { Microscopy } \\
\text { (standard) }\end{array}$} & \multicolumn{2}{|c|}{ RDT (standard) } & \multicolumn{2}{|c|}{ PCR (standard) } & \multicolumn{2}{|c|}{ Microscopy (standard) } & \multicolumn{2}{|c|}{ RDT (standard) } \\
\hline & $+\mathrm{ve}$ & $-\mathrm{ve}$ & $+\mathrm{ve}$ & $-\mathrm{ve}$ & $+\mathrm{ve}$ & $-\mathrm{ve}$ & $+\mathrm{ve}$ & $-\mathrm{ve}$ & $+\mathrm{ve}$ & $-\mathrm{ve}$ & $+\mathrm{ve}$ & $-\mathrm{ve}$ \\
\hline \multicolumn{13}{|l|}{ A. Microscopy } \\
\hline Positive & 125 & 19 & & & 109 & 35 & 30 & 3 & & & 29 & 4 \\
\hline Negative & 185 & 2543 & & & 280 & 2448 & 23 & 394 & & & 47 & 370 \\
\hline Sensitivity $(95 \% \mathrm{CI})$ & \multicolumn{4}{|c|}{$40.3(34.8-46.0)$} & \multicolumn{2}{|c|}{$28.0(23.6-32.8)$} & \multicolumn{4}{|c|}{$56.6(42.3-70.2)$} & \multicolumn{2}{|c|}{$38.2(27.2-50.0)$} \\
\hline Specificity $(95 \% \mathrm{CI})$ & \multicolumn{4}{|c|}{$99.3(98.8-99.6)$} & \multicolumn{2}{|c|}{$98.6(98.0-99.0)$} & \multicolumn{4}{|c|}{$99.2(97.8-99.8)$} & \multicolumn{2}{|c|}{$98.9(97.3-99.7)$} \\
\hline PPV (95\% CI) & \multicolumn{4}{|c|}{$86.8(80.2-91.9)$} & \multicolumn{2}{|c|}{$75.7(67.9-82.4)$} & \multicolumn{4}{|c|}{$90.9(75.7-98.1)$} & \multicolumn{2}{|c|}{$87.9(71.8-96.6)$} \\
\hline NPV (95\% CI) & \multicolumn{4}{|c|}{$93.2(92.2-94.1)$} & \multicolumn{2}{|c|}{89.7 (88.5-90.9) } & \multicolumn{4}{|c|}{$94.5(91.8-96.5)$} & \multicolumn{2}{|c|}{$88.7(85.3-91.6)$} \\
\hline Accuracy $(95 \% \mathrm{CI})$ & \multicolumn{4}{|c|}{$92.9(92.0-93.8)$} & \multicolumn{2}{|c|}{$89.0(87.9-90.2)$} & 94.2 & $.1-96.4)$ & & & 88.7 & .7-91.6) \\
\hline Youden's $J$ index $(95 \% \mathrm{CI})$ & 0.40 & $34-0.46)$ & & & 0.27 & $.22-0.32)$ & 0.56 & $40-0.70)$ & & & 0.37 & $24-0.50)$ \\
\hline B. RDT & & & & & & & & & & & & \\
\hline Positive & 144 & 245 & 109 & 280 & & & 37 & 39 & 29 & 47 & & \\
\hline Negative & 166 & 2317 & 35 & 2448 & & & 16 & 358 & 4 & 370 & & \\
\hline Sensitivity (95\% CI) & 46.5 & $0.8-52.2)$ & 75.7 & $7.9-82.4)$ & & & 69.8 & $.7-81.7)$ & 87.9 & $-96.6)$ & & \\
\hline Specificity $(95 \% \mathrm{CI})$ & 90.4 & $9.2-91.5)$ & 89.7 & $8.5-90.9)$ & & & 90.2 & $.8-92.9)$ & 88.7 & $-91.6)$ & & \\
\hline PPV (95\% CI) & 37.0 & $2.2-42.0)$ & 28.0 & $3.6-32.8)$ & & & 48.7 & $.0-60.4)$ & 38.2 & $-50.0)$ & & \\
\hline NPV (95\% CI) & 93.3 & $2.3-94.3)$ & 98.6 & $8.0-99.0)$ & & & 95.7 & $.1-97.5)$ & 98.9 & $-99.7)$ & & \\
\hline Accuracy $(95 \% \mathrm{CI})$ & 85.7 & $4.4-87.0)$ & 89.0 & 7.9-90.2) & & & 87.8 & $.7-90.8)$ & 88.7 & $-91.6)$ & & \\
\hline Youden's $J$ index $(95 \% \mathrm{CI})$ & 0.37 & $30-0.44)$ & 0.65 & $.56-0.73)$ & & & 0.60 & $42-0.75)$ & 0.77 & $-0.88)$ & & \\
\hline C. PCR & & & & & & & & & & & & \\
\hline Positive & & & 125 & 185 & 144 & 166 & & & 30 & 23 & 37 & 16 \\
\hline Negative & & & 19 & 2543 & 245 & 2317 & & & 3 & 394 & 39 & 358 \\
\hline Sensitivity (95\% CI) & & & 86.8 & $0.2-91.9)$ & 37.0 & $2.2-42.0)$ & & & 90.9 & $-98.1)$ & 48.7 & $.0-60.4)$ \\
\hline Specificity (95\% CI) & & & 93.2 & $2.2-94.1)$ & 93.3 & 2.3-94.3) & & & 94.5 & $-96.5)$ & 95.7 & $.1-97.5)$ \\
\hline PPV $(95 \%$ CI $)$ & & & 40.3 & $4.8-46.0)$ & 46.5 & $0.8-52.2)$ & & & 56.6 & $-70.2)$ & 69.8 & $.7-81.7)$ \\
\hline NPV $(95 \%$ CI $)$ & & & 99.3 & $8.8-99.6)$ & 90.4 & $9.2-91.5)$ & & & 99.2 & $-99.8)$ & 90.2 & $5.8-92.9)$ \\
\hline Accuracy $(95 \% \mathrm{CI})$ & & & 92.9 & $2.0-93.8)$ & 85.7 & $4.4-87.0)$ & & & 94.2 & $-96.4)$ & 87.8 & $.7-90.8)$ \\
\hline Youden's $J$ index $(95 \% \mathrm{CI})$ & & & 0.80 & $.72-0.86)$ & 0.30 & $.24-0.36)$ & & & 0.85 & $-0.95)$ & 0.44 & $30-0.58)$ \\
\hline
\end{tabular}

$P P V$, positive predictive value; $N P V$, negative predictive value; $+v e$, positive; $-v e$, negative

low endemic regions (Fig. $2 \mathrm{a}$ and b). Malaria detection ability of RDT (AUC: 0.68 vs $0.80 ; p=0.001$ ) and microscopy (AUC: 0.70 vs $0.78 ; p=0.029$ ) was lower in asymptomatic than symptomatic participants (Fig. $2 \mathrm{c}$ and d), but found higher in children (AUC: 0.74 vs $0.61 ; p<$ 0.0001 ) in comparison to adults (AUC: 0.76 vs $0.61 ; p<$ 0.0001 ) (Fig. 2 e and f). A total of 118 samples were observed to be positive by all three diagnostic methods. However, 145 samples were positive only by PCR, 2 by microscopy and 264 by RDT. The possible reasons of higher RDT positivity than PCR might be attributed to the persistence of Plasmodium falciparum-encoded HRP2 in the blood even after parasite clearance, which is the target of RDTs (Fig. 3).

\section{Discussion}

The present study highlights the possible setbacks in correctly identifying malaria infection by RDT and microscopy in low and high endemic regions of two North-Eastern districts of India. The study also exemplified the role of possible factors such as age, asymptomatic/symptomatic status of population and malaria-endemic regions in affecting the diagnostic performance of RDT, microscopy and PCR. Most of the crosssectional surveys use microscopy as a comparator to check the diagnostic accuracy of RDTs, due to non-availability of resources and expertise in the field conditions to deploy molecular tools. The present study however is the first large-scale mass blood survey to use molecular diagnosis along with 
Table 4 Comparison of diagnostic accuracy of microscopy, RDT and PCR between children and adults

\begin{tabular}{|c|c|c|c|c|c|c|c|c|c|c|c|c|}
\hline \multirow{3}{*}{ Diagnostics used } & \multicolumn{6}{|c|}{ Children $\leq 14$ years $(n=2106)$} & \multicolumn{6}{|c|}{ Adults above 14 years $(n=1216)$} \\
\hline & \multicolumn{2}{|c|}{ PCR (standard) } & \multicolumn{2}{|c|}{$\begin{array}{l}\text { Microscopy } \\
\text { (standard) }\end{array}$} & \multicolumn{2}{|c|}{ RDT (standard) } & \multicolumn{2}{|c|}{ PCR (standard) } & \multicolumn{2}{|c|}{ Microscopy (standard) } & \multicolumn{2}{|c|}{ RDT (standard) } \\
\hline & $+\mathrm{ve}$ & $-\mathrm{ve}$ & +ve & $-\mathrm{ve}$ & $+\mathrm{ve}$ & $-\mathrm{ve}$ & $+\mathrm{ve}$ & $-\mathrm{ve}$ & $+\mathrm{ve}$ & $-\mathrm{ve}$ & + ve & $-\mathrm{ve}$ \\
\hline \multicolumn{13}{|l|}{ A. Microscopy } \\
\hline Positive & 131 & 18 & & & 121 & 28 & 24 & 4 & & & 17 & 11 \\
\hline Negative & 120 & 1837 & & & 238 & 1719 & 88 & 1100 & & & 89 & 1099 \\
\hline Sensitivity $(95 \% \mathrm{CI})$ & \multicolumn{4}{|c|}{$52.2(45.8-58.5)$} & \multicolumn{2}{|c|}{$33.7(28.8-38.9)$} & \multicolumn{4}{|c|}{$21.4(14.2-30.2)$} & \multicolumn{2}{|c|}{$16.0(9.6-24.4)$} \\
\hline Specificity $(95 \% \mathrm{CI})$ & \multicolumn{4}{|c|}{$99.0(98.5-99.4)$} & \multicolumn{2}{|c|}{$98.4(97.7-98.9)$} & \multicolumn{4}{|c|}{$99.6(99.1-99.9)$} & \multicolumn{2}{|c|}{$99.0(98.2-99.5)$} \\
\hline PPV $(95 \% \mathrm{CI})$ & \multicolumn{4}{|c|}{$87.9(81.6-92.7)$} & \multicolumn{2}{|c|}{$81.2(74.0-87.1)$} & \multicolumn{4}{|c|}{$85.7(67.3-96.0)$} & \multicolumn{2}{|c|}{$60.7(40.6-78.5)$} \\
\hline NPV (95\% CI) & \multicolumn{4}{|c|}{$93.9(92.7-94.9)$} & \multicolumn{2}{|c|}{$87.8(86.3-89.3)$} & \multicolumn{4}{|c|}{$92.6(91.0-94.0)$} & \multicolumn{2}{|c|}{$92.5(90.9-93.9)$} \\
\hline Accuracy $(95 \% \mathrm{CI})$ & \multicolumn{4}{|c|}{$93.4(92.4-94.5)$} & \multicolumn{2}{|c|}{$87.4(85.9-88.8)$} & 92.4 & 0.9-93.9) & & & 91.8 & $0.2-93.3)$ \\
\hline Youden's $J$ index $(95 \% \mathrm{CI})$ & 0.51( & $44-0.58)$ & & & 0.32 & $.26-0.38)$ & 0.21 & $.13-0.30)$ & & & 0.15 & $.08-0.24)$ \\
\hline B. RDT & & & & & & & & & & & & \\
\hline Positive & 148 & 211 & 121 & 238 & & & 33 & 73 & 17 & 89 & & \\
\hline Negative & 103 & 1644 & 28 & 1719 & & & 79 & 1031 & 11 & 1099 & & \\
\hline Sensitivity $(95 \% \mathrm{CI})$ & 59.0 & $2.6-65.1)$ & 81.2 & $4.0-87.1)$ & & & 29.5 & $1.2-38.8)$ & 60.7 & $-78.5)$ & & \\
\hline Specificity $(95 \% \mathrm{CI})$ & 88.6 & 7.1-90.0) & 87.8 & $6.3-89.3)$ & & & 93.4 & $1.8-94.8)$ & 92.5 & $-93.9)$ & & \\
\hline PPV (95\% CI) & 41.2( & $6.1-46.5)$ & 33.7 & $8.8-38.9)$ & & & 31.1 & $2.5-40.9)$ & 16.0 & 24.4) & & \\
\hline NPV $(95 \%$ CI) & 94.1( & 2.9-95.2) & 98.4 & 7.7-98.9) & & & 92.9 & $1.2-94.3)$ & 99.0 & $-99.5)$ & & \\
\hline Accuracy $(95 \% \mathrm{CI})$ & 85.1( & $3.6-86.6)$ & 87.4 & $5.9-88.8)$ & & & 87.5 & $5.6-89.4)$ & 91.8 & $-93.3)$ & & \\
\hline Youden's $J$ index $(95 \% \mathrm{CI})$ & 0.48 & $40-0.55)$ & 0.69 & $.60-0.76)$ & & & 0.23 & $.13-0.34)$ & 0.53 & $-0.72)$ & & \\
\hline C. PCR & & & & & & & & & & & & \\
\hline Positive & & & 131 & 120 & 148 & 103 & & & 24 & 88 & 33 & 79 \\
\hline Negative & & & 18 & 1837 & 211 & 1644 & & & 4 & 1100 & 73 & 1031 \\
\hline Sensitivity (95\% CI) & & & 87.9 & $1.6-92.7)$ & 41.2 & $6.1-46.5)$ & & & 85.7 & $-96.0)$ & 31.1 & $2.5-40.9)$ \\
\hline Specificity $(95 \%$ CI) & & & 93.9 & 2.7-94.9) & 94.1 & 2.9-95.2) & & & 92.6 & $-94.0)$ & 92.9 & $1.2-94.3)$ \\
\hline PPV $(95 \%$ CI $)$ & & & 52.2 & $5.8-58.5)$ & 59.0 & $2.6-65.1)$ & & & 21.4 & $-30.2)$ & 29.5 & $1.2-38.8)$ \\
\hline NPV (95\% CI) & & & 99.0 & $8.5-99.4)$ & 88.6 & 7.1-90.0) & & & 99.6 & -99.9) & 93.4 & $1.8-94.8)$ \\
\hline Accuracy $(95 \% \mathrm{CI})$ & & & 93.4 & $2.4-94.5)$ & 85.1 & $3.6-86.6)$ & & & 92.4 & $-93.9)$ & 87.5 & $5.6-89.4)$ \\
\hline Youden's $J$ index $(95 \% \mathrm{CI})$ & & & 0.82 & $.74-0.88)$ & 0.35 & $.29-0.42)$ & & & 0.78 & $-0.90)$ & 0.24 & $14-0.35)$ \\
\hline
\end{tabular}

$P P V$, positive predictive value; $N P V$, negative predictive value; $+v e$, positive; $-v e$, negative

standard diagnostic tools in the selected North-Eastern regions of India.

Here we report that RDT in comparison to microscopy had higher sensitivity but substantially lower specificity and PPV when PCR was used as a reference standard. These observations suggest that RDT despite having better performance than microscopy may not be useful in accurately determining the proportion of individuals without malaria as malaria negative (due to low specificity). Furthermore, there was very low probability of correctly identifying people as RDT positives among those who had malaria, as reflected by its low PPV. These explanations limit the usefulness of RDTs in mass blood surveys (Parker et al. 2016) conducted in nontransmission season; nevertheless, such measures depend upon the comparator or reference standard used while doing the analysis (Bell et al. 2005). Low density of parasites in individuals might be the plausible reasons for low PPV of RDT. For instance, PCR, like RDT, also showed lower specificity and PPV than microscopy because of inherent inaccuracies associated with RDTs to be used as a reference standard. In low endemic regions, the performance of diagnostic tools found lower than it was observed in high endemic regions. Most of the scientific reports evidenced low parasite density as the prime cause of low performance of standard diagnostic tools (Abeku et al. 2008). Also, it is known that measures such as sensitivity and PPV are proportionate to the disease prevalence (Trevethan 2017) as seen in this study where sensitivity and PPV was higher in high endemic regions. We found high false positive rate (FPR) in RDT than microscopy irrespective of endemic regions. Especially, FPR was much more in high 

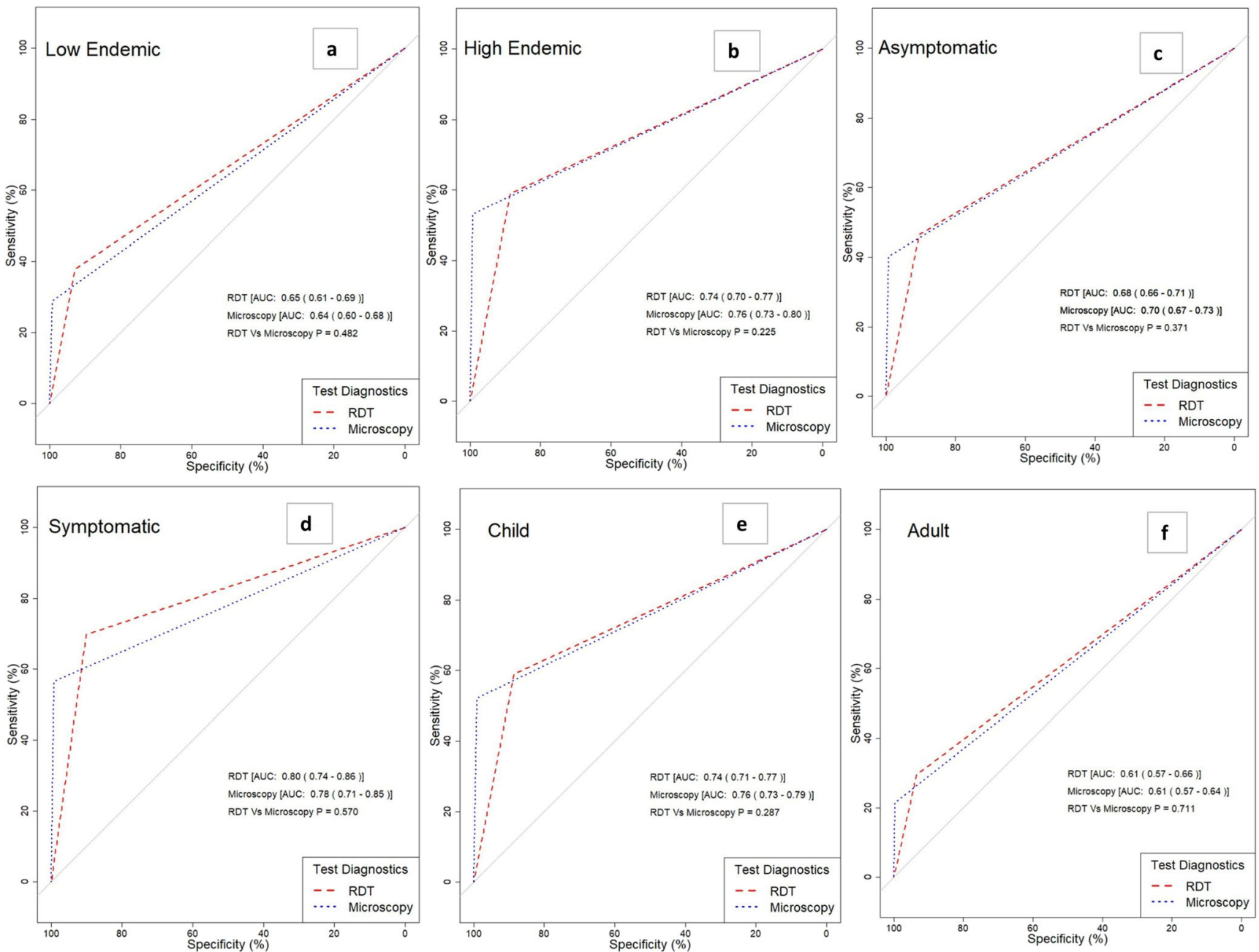

Fig. 2 ROC curve analysis considering PCR as a reference standard. Panel $\mathbf{a}$ and $\mathbf{b}$ : Comparison of AUC with endemicity (low vs high): RDT $(p=0.0014)$; microscopy $(p<0.0001)$. Panel $\mathbf{c}$ and $\mathbf{d}$ : Comparison of AUC with symptoms (asymptomatic vs symptomatic):

compared to low endemic regions (Abeku et al. 2008) when PCR was used as reference standard. Contrary to this, false negative rate (FNR) was found to be higher in microscopy than RDT irrespective of endemic regions and even higher in low compared to high endemic regions. FPR and FNR both are the major concerns for public health as FPR on one hand exposes healthy individual by unnecessary administration of drugs and creates drug pressure on parasites, thereby could result in drug resistance. While on the other hand, high FNR renders a certain proportion of population to devoid of accurate diagnosis and correct treatment. High FNR observed in our study might be associated with the presence of deletion in Pfhrp 2/3 gene of Plasmodium falciparum, which is reported near to $25 \%$ in Indian settings (Bharti et al. 2016). This could have serious implications in disease transmission that may also result in mortality (Berzosa et al. 2018). Interestingly, RDT performance was at par with PCR in low endemic regions, when data were analysed using microscopy as a
RDT ( $p=0.0013)$; microscopy $(p=0.029)$. Panel e and $\mathbf{f}$ : Comparison of AUC with age group (child vs adult): RDT $(p<0.0001)$; microscopy ( $p$ $<0.0001)$

reference standard; nevertheless, this was not the case in high endemic regions. These observations suggest promising use of RDTs in low transmission settings, but caution should be taken in deploying these RDTs to precisely detect malaria infection, due to the variabilities associated with its sensitivity and specificity between different areas, seasons and age groups (Abeku et al. 2008).

Our data suggest better malaria detection ability of microscopy and RDT in symptomatic compared to asymptomatic individuals. In comparison to symptomatic where standard diagnostic tools are losing nearly $30-40 \%$ malaria cases, approximately $60 \%$ malaria positive cases were missed among asymptomatic individuals. On the other hand, only 9\% symptomatic and $13 \%$ asymptomatic cases could not be detected by PCR when microscopy was used as a reference. These results are in agreement to the view that microscopy and RDT are able to detect nearly all symptomatic infections but advanced molecular techniques are needed for the detection of asymptomatic infections in low 
Fig. 3 Venn diagram showing positive results using three different malaria diagnostic methods. A total of 118 samples are showing concordance in overlapping section, when detected using three different techniques

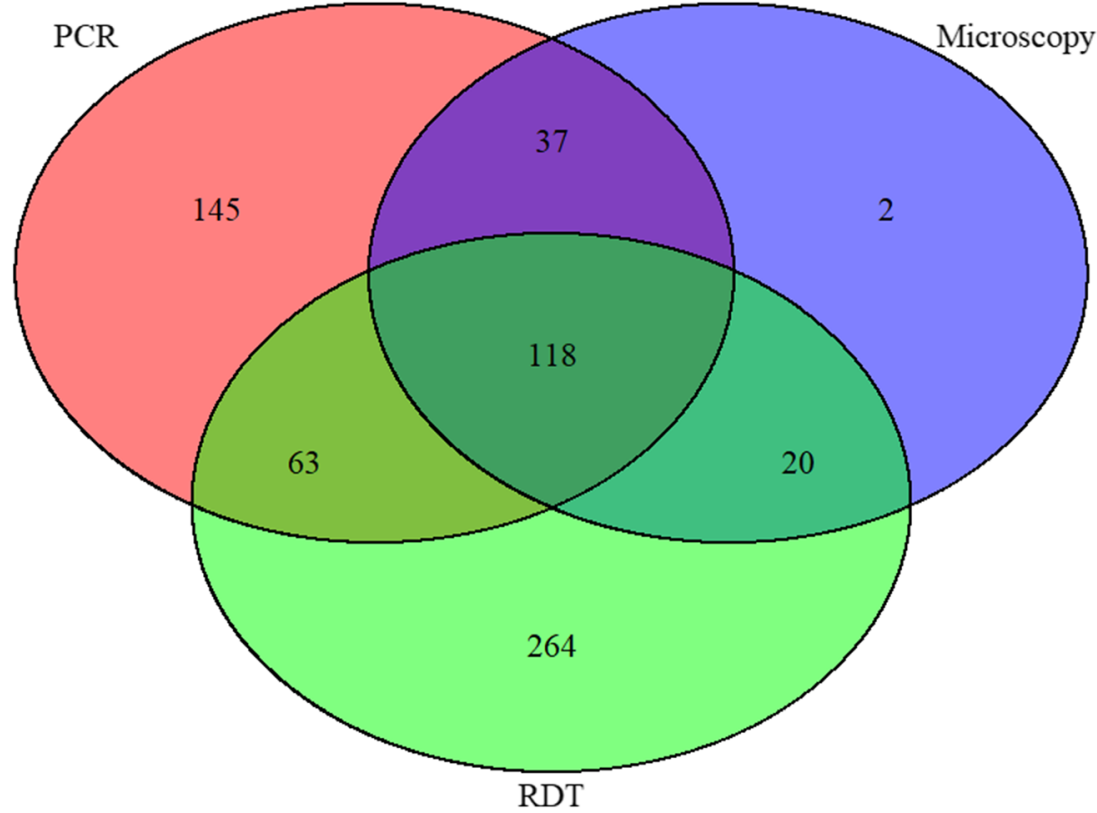

transmission settings (Turki et al. 2015) thus limit the use of RDTs in population level surveillance for estimating true malaria burden. The fraction of low-density parasitaemia in patent and sub-patent infections is associated with the parasite as well as host factors, and investigations also suggest that high proportion of low-density infections that remained undetected by microscopy and RDT in low malaria-endemic region is asymptomatic (Nicastri et al. 2009; Harris et al. 2010). Nevertheless, the density of parasites in asymptomatic individuals never remains fixed; therefore, it needs to be checked periodically to optimise the utility of RDT in varying transmission settings (McMorrow et al. 2011). We found a dramatic change in sensitivity of microscopy and RDT when used for malaria screening between children and adults. Children have lower immunity than adults, which may be attributable to higher sensitivity of conventional malaria diagnostic methods in children than adults, as observed in our study. A recent meta-analysis on assessing the ability of RDTs in malaria diagnosis supports our findings about high accuracy of RDT in children, especially in those children residing in high transmission regions (Zhu et al. 2020). The adults encounter higher number of infection episodes in their life and therefore present low-density infections due to higher interference of antibodies developed against encountered pathogen (Osei-Yeboah et al. 2016). The ROC curve analysis was used to calculate AUC that ranged between 0.5 and 1 , where 0.5 is unacceptable test and 1 is outstanding. AUC values of RDT and microscopy in the range of 0.74 to 0.76 suggest carefully using these conventional diagnostic methods in mass blood surveys. AUC data suggest that RDT and microscopy in comparison to PCR were not much effective in discriminating malaria-positive and -negative individuals in low malaria-endemic areas, adults and asymptomatic cases.
Precisely, RDT positivity reflects the plasmodium antigenaemia, not parasitaemia. Therefore, proportion of RDT positive population reflects presence of malaria antigen in their blood. It does not reflect the prevalence of parasite, which can be estimated by microscopy and PCR. In our study, out of total positive cases detected with either diagnostic method $(N=649)$, more than $40 \%(264 / 649)$ were detected exclusively by RDT and not by higher sensitive technique like PCR. This might be due to the persistence of plasmodium antigenaemia such as ongoing HRP2 circulation in recently treated infections, sub-microscopic infection in the community and human errors related to the interpretation or recording of RDT results (McMorrow et al. 2011).

\section{Conclusion}

Our results suggest that RDT in comparison to microscopy has high sensitivity but low specificity and PPV. Areaspecific usage of standard diagnostic tools along with advanced malaria detection methods should be envisaged. Nevertheless, active case detection using RDT in mass blood surveys may be used, specifically in low endemic regions. Thus, low endemic regions, asymptomatic malaria cases and adult age group are to be dealt with extra caution while using standard malaria diagnosis tools.

Acknowledgements The guidance and support provided by Dr. William Layam, Sr. Regional Director Meghalaya, Dr. Parthajyoti Gogoi, Sr. Regional Director Assam, Dr. H. P. Gupta and Dr. Vas Dev, ExOfficer-in-Charge, NIMR field unit, Guwahati is highly acknowledged. We thank State Health Programme and Dr. Neena Valecha, Director, 
NIMR for being a coordinator of the study. The authors also wish to extend their thanks to the experienced technicians of our institute for performing rigorous quality check of microscopy results.

Author contribution SP was the principal investigator of the study. SP and NM conceived and designed the study. HS and MPS contributed substantially in data collection, data analysis and interpretation of the results. HS, SP and MPS executed the field and laboratory work. KS and NM coordinated the field operations and logistic arrangements. HS drafted the first version of the manuscript. MPS and NM critically reviewed the manuscript and all authors provided intellectual inputs, reviewed and approved this manuscript.

Funding This study was funded by the Indian Council of Medical Research, New Delhi, India vide grant no. NER/55/2015-ECD-I.

Data availability All relevant data is available in the manuscript.

Code availability Not applicable

\section{Declarations}

Ethical approval The procedures performed in this study were in accordance with the ethical standards of the Institutional Ethics Committee of National Institute of Malaria Research (Ref. No. ECR/ NIMR/ EC/ 2015/ 490). Informed consent was obtained from all individual participants included in the study.

Conflict of interest The authors declare/s no competing interests.

\section{References}

Abeku TA, Kristan M, Jones C, Beard J, Mueller DH, Okia M, Rapuoda B, Greenwood B, Cox J (2008) Determinants of the accuracy of rapid diagnostic tests in malaria case management: evidence from low and moderate transmission settings in the East African highlands. Malar J 7:202. https://doi.org/10.1186/1475-2875-7-202

Bell DR, Wilson DW, Martin LB (2005) False-positive results of a Plasmodium falciparum histidine-rich protein 2-detecting malaria rapid diagnostic test due to high sensitivity in a community with fluctuating low parasite density. Am J Trop Med Hyg 73:199203. https://doi.org/10.4269/ajtmh.2005.73.199

Berzosa P, de Lucio A, Romay-Barja M, Herrador Z, González V, García L, Fernández-Martínez A, Santana-Morales M, Ncogo P, Valladares B, Riloha M, Benito A (2018) Comparison of three diagnostic methods (microscopy, RDT, and PCR) for the detection of malaria parasites in representative samples from Equatorial Guinea. Malar J 17:333. https://doi.org/10.1186/s12936-018-2481-4

Bharti PK, Chandel HS, Ahmad A, Krishna S, Udhayakumar V, Singh N (2016) Prevalence of pfhrp2 and/or pfhrp3 gene deletion in plasmodium falciparum population in eight highly endemic states in India. PLoS One 11:e0157949. https://doi.org/10.1371/journal.pone. 0157949

Chanda P, Castillo-Riquelme M, Masiye F (2009) Cost-effectiveness analysis of the available strategies for diagnosing malaria in outpatient clinics in Zambia. Cost Eff Resour Alloc 7:5. https://doi.org/10. 1186/1478-7547-7-5

Harris I, Sharrock WW, Bain LM, Gray KA, Bobogare A, Boaz L, Lilley K, Krause D, Vallely A, Johnson ML, Gatton ML, Shanks G, Cheng Q (2010) A large proportion of asymptomatic Plasmodium infections with low and sub-microscopic parasite densities in the low transmission setting of Temotu Province, Solomon Islands: challenges for malaria diagnostics in an elimination setting. Malar J 9: 254. https://doi.org/10.1186/1475-2875-9-254

Kaura T, Kaur J, Sharma A, Dhiman A, Pangotra M, Upadhyay AK, Grover GS, Sharma SK (2019) Prevalence of submicroscopic malaria in low transmission state of Punjab: a potential threat to malaria elimination. J Vector Borne Dis 56:78-84. https://doi.org/10.4103/ 0972-9062.257780

McMorrow ML, Aidoo M, Kachur SP (2011) Malaria rapid diagnostic tests in elimination settings - can they find the last parasite? Clin Microbiol Infect 17:1624-1631. https://doi.org/10.1111/j.14690691.2011.03639.x

Ministry of Health \& Family Welfare, Government of India (2016) National framework for malaria elimination In India (2016-2030). https://apps.who.int/iris/bitstream/handle/10665/246096/national_ framework_malaria_elimination_india_2016_2030.pdf? sequence= 1\&isAllowed=y. Accessed 23 Aüg $20 \overline{20}$

National Vector Borne Disease Control Programme (2019) Magnitude of the problem. https://nvbdcp.gov.in/index4.php?lang=1\&level= 0\&linkid $=420 \&$ lid $=3699$. Accessed 23 Aug 2020

Nicastri E, Bevilacqua N, Sañé Schepisi M et al (2009) Accuracy of malaria diagnosis by microscopy, rapid diagnostic test, and PCR methods and evidence of antimalarial overprescription in nonsevere febrile patients in two Tanzanian hospitals. Am J Trop Med Hyg 80:712-717

Osei-Yeboah J, Kwame Norgbe G, Yao Lokpo S, et al. (2016) Comparative performance evaluation of routine malaria diagnosis at Ho Municipal Hospital. J Parasitol Res Article ID: 5837890. https://www.hindawi.com/journals/jpr/2016/5837890/

Parker DM, Landier J, von Seidlein L, Dondorp A, White L, Hanboonkunupakarn B, Maude RJ, Nosten FH (2016) Limitations of malaria reactive case detection in an area of low and unstable transmission on the Myanmar-Thailand border. Malar J 15:571. https://doi.org/10.1186/s12936-016-1631-9

QIAGEN (2020) QIAamp DNA Mini and Blood Mini Handbook. https:// www.qiagen.com/us/resources/resourcedetail id=62a200d6-faf4469b-b50f-2b59cf738962\&lang=en. Accessed 23 Aug 2020

Rafael ME, Taylor T, Magill A, Lim YW, Girosi F, Allan R (2006) Reducing the burden of childhood malaria in Africa: the role of improved. Nature 444(Suppl 1):39-48. https://doi.org/10.1038/ nature 05445

Shankar H, Phookan S, Singh MP, Bharti RS, Ahmed N, Yadav CP, Sharma GP, Singh K, Kaur H, Mishra N (2021). Asymptomatic low-density Plasmodium infection during non-transmission season: a community-based cross-sectional study in two districts of North Eastern Region, India. Trans R Soc Trop Med Hyg. https://doi.org/ 10.1093/trstmh/trab017

Snounou G, Viriyakosol S, Jarra W, Thaithong S, Brown KN (1993) Identification of the four human malaria parasite species in field samples by the polymerase chain reaction and detection of a high prevalence of mixed infections. Mol Biochem Parasitol 58:283-292. https://doi.org/10.1016/0166-6851(93)90050-8

Trevethan R (2017) Sensitivity, specificity, and predictive values: foundations, pliabilities, and pitfalls in research and practice. Front Public Health 5:307. https://doi.org/10.3389/fpubh.2017.00307

Turki H, Raeisi A, Malekzadeh K, Ghanbarnejad A, Zoghi S, Yeryan M, Abedi Nejad M, Mohseni F, Shekari M (2015) Efficiency of nestedPCR in detecting asymptomatic cases toward malaria elimination program in an endemic area of Iran. Iran J Parasitol 10:39-45

Uzochukwu BS, Obikeze EN, Onwujekwe OE et al (2009) Costeffectiveness analysis of rapid diagnostic test, microscopy and syndromic approach in the diagnosis of malaria in Nigeria: implications for scaling-up deployment of ACT. Malar J 8:265. https://doi. org/10.1186/1475-2875-8-265

van Eijk AM, Sutton PL, Ramanathapuram L, Sullivan SA, Kanagaraj D, Priya GSL, Ravishankaran S, Asokan A, Sangeetha V, Rao PN, 
Wassmer SC, Tandel N, Patel A, Desai N, Choubey S, Ali SZ, Barla P, Oraon RR, Mohanty S, Mishra S, Kale S, Bandyopadhyay N, Mallick PK, Huck J, Valecha N, Singh OP, Pradhan K, Singh R, Sharma SK, Srivastava HC, Carlton JM, Eapen A (2019) The burden of submicroscopic and asymptomatic malaria in India revealed from epidemiology studies at three varied transmission sites in India. Sci Rep 9:17095. https://doi.org/10.1038/s41598-019-53386-w

van Eijk AM, Mannan AS, Sullivan SA, Carlton JM (2020) Defining symptoms of malaria in India in an era of asymptomatic infections. Malar J 19:237. https://doi.org/10.1186/s12936-020-03310-9

Wongsrichanalai C, Barcus MJ, Muth S et al (2007) A review of malaria diagnostic tools: microscopy and rapid diagnostic test (RDT). Am J
Trop Med Hyg 77:119-127. https://doi.org/10.4269/ajtmh.2007.77. 119

World Health Organization (2019) World malaria report 2019. https:// www.who.int/news-room/feature-stories/detail/world-malariareport-2019. Accessed 23 Aug 2020

Zhu W, Ling X, Shang W, du Y, Liu J, Cao Y, Yang M, Zhu G, Cao J, Huang $J$ (2020) High value of rapid diagnostic tests to diagnose malaria within children: A systematic review and meta-analysis. J Glob Health 10:010411. https://doi.org/10.7189/jogh.10.010411

Publisher's note Springer Nature remains neutral with regard to jurisdictional claims in published maps and institutional affiliations. 